\title{
Nonlinear Optics Applications
}

\author{
Mirosław A. Karpierz ${ }^{* 1}$ and Gaetano Assanto ${ }^{2}$ \\ ${ }^{1}$ Faculty of Physics, Warsaw University of Technology, Koszykowa 75, 00-662 Warszawa \\ ${ }^{2}$ NooEL-Nonlinear Optics and OptoElectronics Lab, University "Roma Tre”, 00146 Rome-Italy
}

Received March 30, 2012; accepted March 31, 2012; published March 31, 2012

\begin{abstract}
This is a brief editorial overview of the work devoted to nonlinear optics in the current issue.
\end{abstract}

The first ten letters of this special issue were prepared by participants to the XI International Workshop on Nonlinear Optics Applications held in Torun, Poland in September 2011. These International Workshops have been organised since 1992 by researchers at the Warsaw University of Technology and the West Pomeranian University of Technology. The Workshops have dealt with experimental and theoretical aspects of the applications of nonlinear optics, but also with the related photonic phenomena. In the last Workshop in Torun there were almost 30 participants from USA, Canada, Germany, France, Sweden, Romania, Finland, Italy and Poland (some of them are in Fig. 1).

The work reported here covers a spectrum of nonlinear optics and its applications: nematicons, i.e. spatial solitons in nematic liquid crystals (G. Assanto et al. and by F. A. Sala and M. A. Karpierz), spectral phase interferometry for direct electric-field reconstruction
(SPIDER) using four wave mixing (A. Pasquazi et al.), self-focusing in Kerr media with both linear and circular birefringence (K. A. Rutkowska et al.), theoretical calculations of third order nonlinearity (A. Karakas et al.), measurements of nematic liquid crystals parameters by nonlinear optical methods (B. W. Klus et al.), modeling of photorefractive multiple quantum well waveguides (E. Weinert-Rączka and M. Wichtowski), paraxial geometrical optics for nonlinear beam propagation ( $\mathrm{P}$. Berczyński and Yu. A. Kravtsov), spatio-temporal pulses in cubic-quintic Kerr like nonlinear media (J. Jasiński). Finally, the issue contains also a report on fluorescence measurement of hybrid nanostructures composed of lightharvesting complex LH2 from purple bacteria and gold nanorods (L. Bujak et al.).

The variety of contents in the current issue shows that nonlinear optics is still quite a vibrant field [1].

\section{References}

[1] M. A. Karpierz and G. I. Stegeman, Nonlinear Optics: A Vibrant Field, Photon. Lett. Poland 1, 145-147 (2009).

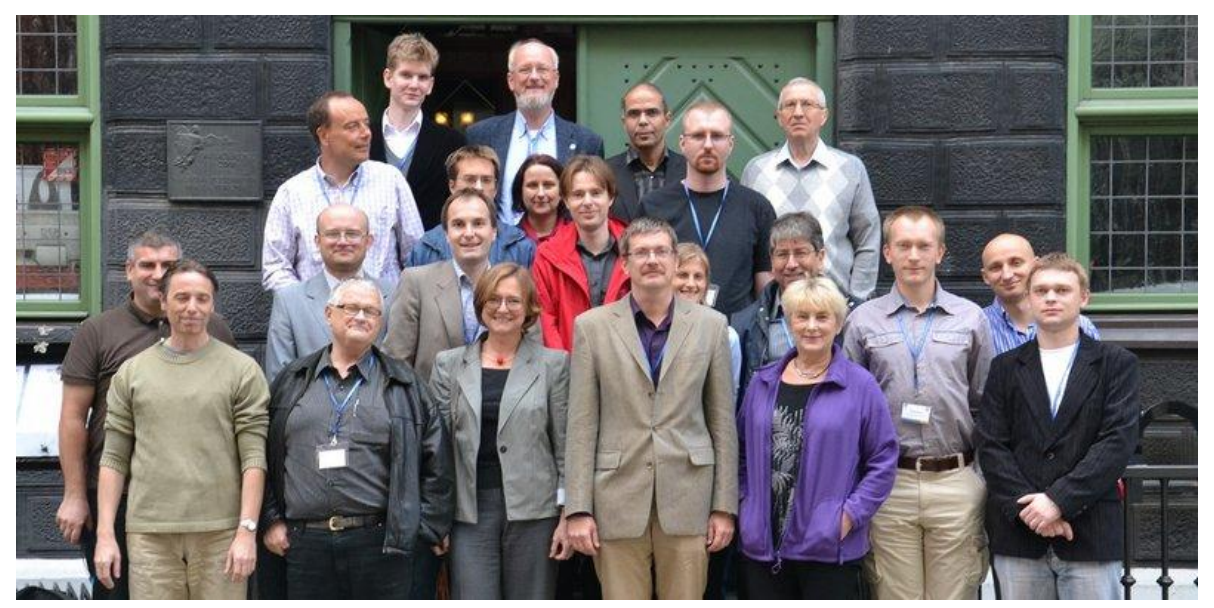

Fig. 1. Participants to the XI International Workshop on Nonlinear Optics Applications (September 2011, Torun, Poland)

*E-mail: karpierz@if.pw.edu.pl 